\title{
Equivariant configuration spaces
}

\author{
Colin Rourke \\ BRIAN SANDERSON \\ Mathematics Institute \\ University of Warwick \\ Coventry CV4 7AL UK \\ Email: cpr@maths.warwick.ac.uk and bjs@maths.warwick.ac.uk
}

\begin{abstract}
We use the compression theorem (cf [7; section 7]) to prove results for equivariant configuration spaces analogous to the well-known non-equivariant results of May, Milgram and Segal $[5,6,8]$.
\end{abstract}

AMS Classification 55P91, 55P35, 55P40; 57R91, 55P45, 55P47

Keywords Equivariant homotopy theory, configuration spaces, multiple loop spaces, multiple suspension, group completion

This paper is concerned with the homotopy type of equivariant configuration spaces. Some of the results proved here are known or implicit in known results $[2,4,8,9]$. However there does not appear to be a systematic treatment in full generality in the literature. Moreover the treatment given here is new and several of our results are deduced from the compression theorem [7] by short, elementary arguments. (The proof of the compression theorem in [7] is also short and elementary.)

We are grateful to Peter May for pointing out this gap in the literature and suggesting that the compression theorem might be used to fill it and we are grateful to Graeme Segal for a copy of his preprint [9]. We would also like to thank the referee for many helpful comments which have resulted in a clearer exposition of our material.

Throughout $G$ is a finite group and $V$ is a finite-dimensional real representation of $G$.

\section{Notation and results}

A based $G$-space $X$ is a $G$-space with basepoint $*$ which is fixed under the action. We shall assume that all base points are non-degenerate. For a $G$-space this means that the inclusion $\{*\} \subset X$ satisfies the HEP through equivariant maps (or equivalently that there is an equivariant retraction of $X \times I$ on $X \times$ $\{0\} \cup\{*\} \times I)$. 
Let $x \in X$, then $G_{x}$ denotes the isotropy group of $x$ in other words the subgroup $\{g \in G \mid g(x)=x\}$ of $G$. Let $H$ be any subgroup of $G$, then $X^{H}:=\left\{x \in X \mid G_{x} \supset H\right\}$ (the fixed-point set of $H$ ) and $X_{H}:=\{x \in X \mid$ $\left.G_{x}=H\right\}$. We say that $X$ is $G$-connected if $X^{H}$ is connected for each subgroup $H$ of $G$.

We denote by $S^{V}$ the $G$-manifold which is the 1-point compactification of $V$ (thought of as Euclidean space with $G$ action) with basepoint at $\infty$. If $X$ is a based $G$-space then $\Omega^{V}(X)$ is the space of based $G$-equivariant maps $S^{V} \rightarrow X$ and $S^{V}(X)$ is the $G$-space $S^{V} \wedge X$ with product action.

Let $M$ be a $G$-manifold without boundary. Let $C_{M}(X)$ denote the space of $G$-equivariant configurations in $M$ with labels in a based $G$-space $X$. Thus a point of $C_{M}(X)$ is a finite subset of $M$ which is closed under the action of $G$ and is equivariantly labelled in $X$. The basepoint of $C_{M}(X)$ is the empty configuration and the topology is such that points labelled by $*$ can be deleted. More precisely, a labelled configuration of $q$ points is a point of the quotient of the $q$-fold cartesian product $\times_{q}(M \times X)$ by the symmetric group $\Sigma_{q}$, acting by diagonal action, such that the $q$ points of $M$ are distinct. The configuration is equivariant if whenever $(m, x)$ is one of the labelled points then so is $(g(m), g(x))$ for all $g \in G$. We identify configurations which agree apart from points labelled by $*$. The topology on $C_{M}(X)$ is the quotient topology. In other words $C_{M}(X)$ is a quotient space of a subset of $\coprod_{q}\left(\times_{q}(M \times X)\right)$.

There is a well-known map $j_{V}: C_{V}(X) \rightarrow \Omega^{V} S^{V}(X)$ which can be described by thinking of the configuration as a set of charged particles and using the electric field they generate (see [8; page 213]). There is a more useful description of $j_{V}$ given by replacing $C_{M}(X)$ by the space $C_{M}^{\circ}(X)$ of "little discs" in $M$ labelled in $X$. To be precise, suppose that $M$ is equipped with an equivariant metric. A little disc at $x \in M$ is an $\varepsilon$-disc $D_{\varepsilon}$ with centre $x$ where $\varepsilon$ is small enough that the exponential map is a diffeomorphism from the $\varepsilon$-disc in the fibre of $T(M)$ at $x$ to $D_{\varepsilon}$. A point of the little disc space $C_{M}^{\circ}(X)$ is a finite disjoint collection of little discs in $M$ which is closed under the action of $G$ and such that each little disc is labelled by a point of $X$ and this labelling is equivariant. The topology is such that discs labelled by $*$ are ignored (as above). The map $C_{M}^{\circ}(X) \rightarrow C_{M}(X)$ which takes each disc to its centre is well-known to be a homotopy equivalence, see eg [3; Appendix on page 289].

Replacing $C_{M}(X)$ by $C_{M}^{\circ}(X)$, the map $j_{V}$ can be described as follows. A little disc at $x \in V$ can be canonically identified with the unit disc $D$ in $V$ (via translation and radial expansion). Given a collection of little discs in $V$ define a map $S^{V} \rightarrow S^{V}$ by mapping each little disc to $S^{V}$ by using the identification with $D$ and the standard identification $D / \partial D=S^{V}$. Map the rest of $V$ to $*$. 
Theorem 1 Let $X$ be a based $G$-connected $G$-space. Then $j_{V}: C_{V}(X) \rightarrow$ $\Omega^{V} S^{V}(X)$ is a weak homotopy equivalence.

Notice that in theorem 1 neither $C_{V}(X)$ nor $\Omega^{V} S^{V}(X)$ is necessarily a monoid. However if we let $V+1$ denote the representation $V \oplus \operatorname{id}_{\mathbb{R}}$ then $C_{V+1}(X)$ is equivalent to a monoid and we can form the classifying space $B C_{V+1}(X)$.

Theorem 2 Let $X$ be a based $G$-space. Then $j_{V+1}$ induces a weak homotopy equivalence $B C_{V+1}(X) \rightarrow \Omega^{V} S^{V+1}(X)$.

Note that in theorem 2 and the following corollary $X$ is not assumed to be $G$ connected. Recall that if $A$ is a topological monoid then the natural map $A \rightarrow$ $\Omega B A$ is called the group completion of $A$. Group completion is a homotopy notion; thus we also refer to a group completion composed with a homotopy equivalence as a group completion. A group completion composed with a weak homotopy equivalence is called a weak group completion (cf remark 1 at the end of this section).

Corollary Let $W$ be a representation with a trivial summand. Then $j_{W}: C_{W}(X) \rightarrow \Omega^{W} S^{W}(X)$ is a weak group completion.

Proof Write $W=V+1$ and apply theorem 2 .

Theorem 1 is new. The special case when $V$ has a trivial summand and the corollary to theorem 2 are proved in Hauschild [2] (Hauschild proves the result for $X=S^{0}$, however his methods extend with little alteration, see the remarks at the end of [2]). A generalisation of theorem 1 (where $V$ is replaced by an arbitrary $G$-manifold) is given in section 4 . Theorem 2 can be deduced using Hauschild's methods [2], but we prefer instead to deduce it using Segal's methods [8], which can be adapted to prove the following stronger result:

Theorem 3 Let $M$ be a $G$-manifold. Then $B C_{M \times \mathbb{R}}(X)$ is homotopy equivalent to $C_{M}(S X)$.

In the case that $X$ is not necessarily $G$-connected and $V$ has no trivial summand, then the above results give us no information about $C_{V}(X)$. However in this case we can write $C_{V}(X)$ as $X^{G} \times A$ where $A$ is a certain topological monoid (for details here see section 4 ). We then have:

Theorem 4 There is a weak homotopy equivalence $t: \Omega^{V} S^{V}(X) \rightarrow X^{G} \times$ $\Omega B A$ such that $t \circ j_{V}$ is homotopic to the natural map $C_{V}(X)=X^{G} \times A \rightarrow$ $X^{G} \times \Omega B A$. 
This result improves on Segal's result [9; theorem B]. Segal proves a stable result for the special case $X=S^{0}$. Theorem 4 is significantly sharper even in this special case.

Remarks 1 We have been careful to state exactly when we can prove homotopy equivalence and when we can only prove weak homotopy equivalence. It is worth noting that if $X$ has the $G$-homotopy type of a $G$-CW complex then all the spaces defined above have the homotopy type of CW complexes; in this case the word "weak" can be deleted from all the results (cf Araki-Murayama [1] and Waner [10]).

2 As remarked below theorem 1, equivariant configuration spaces and loopssuspension spaces are not usually monoids. This contrasts with the situation in the classical loops-suspension theorem where the configuration space approximates the monoid structure closely (see eg May [5]). The one case where the configuration space $C_{V}(X)$ is a monoid (up to homotopy) is when the representation $V$ has a trivial summand. In this case $\Omega^{V} S^{V}(X)=\Omega\left(\Omega^{V^{\prime}} S^{V}(X)\right)$ is a loop space (where $V=V^{\prime}+1$ ). The equivalent monoid structure on $C_{V}(X)$ is described in detail in section 3 (see the start of the description of $q$ ) and this description makes it clear that $j_{V}$ commutes (up to homotopy) with the monoid structures. This fact is implicit in theorem 2 .

\section{Proof of theorem 1}

We follow the proof given in [7] of the non-equivariant case. Here is a quick sketch of the argument. A representative of $\pi_{k} C_{V}(X)$ is a parallel framed manifold in $\mathbb{R}^{k} \times V$ equivariantly labelled in $X$, whilst a representative of $\pi_{k} \Omega^{V} S^{V}(X)$ is a semi-parallel framed manifold. The compression theorem applied locally implies that they are equivalent. The description of $\pi_{k} \Omega^{V} S^{V}(X)$ is justified by transversality to $0 \in V$. The concepts "parallel" and "semiparallel" are defined in the detailed version of the argument, which now follows. We start with the details of the transversality result that we need.

Consider a $G$-map $f: V \times Q \rightarrow V \times X$, where $Q$ is a smooth manifold with trivial $G$-action, such that the composition with projection on $V$ is smooth. Let $x \in V \times Q$ and let $H=G_{x}$ (the isotropy group of $x$ ) and let $W=V^{H}$ (the subspace of $V$ fixed by $H$ ). So $x \in W \times Q$. Let $U$ be the orthogonal complement of $W$ in $V$ (we assume without loss that we have an equivariant Riemannian metric on all $G$-manifolds) then $U$ can be identified with each fibre of the normal bundle of $W \times Q$ in $V \times Q$. Denote the fibre at $x$ by $U_{x}$. 
Now $f(x) \in W \times X$ and we can again identify $U$ with each fibre of the normal bundle of $W \times X$ in $V \times X$. Denote the fibre at $f(x)$ by $U_{f x}$. We say that $f$ is semi-parallel at $x$ if there is a neighbourhood $N$ of 0 in $U$ such that $f \mid N_{x}$ is the identity $N_{x} \rightarrow N_{f x}$, where $N_{x}, N_{f x}$ are the copies of $N$ in $U_{x}, U_{f x}$ respectively. There is a similar definition of semi-parallel near a subset of $V_{H} \times Q$ which is closed in $W \times Q$. (Recall that $V_{H} \subset W=V^{H}$ is the subset of points with isotropy group $H$.)

We say that $f$ is transverse to $0 \times X \subset V \times X$ if for each subgroup $H$ of $G$ we have that $f \mid V_{H} \times Q$ is transverse to $0 \times X \subset V \times X$ and further $f^{-1}(0 \times X) \cap V_{H} \times$ $Q$ is a closed subset $C$ of $V^{H} \times Q$ (in fact a submanifold by transversality) and $f$ is semi-parallel near $C$. (Notice that the definition implies that $f^{-1}(0 \times X)$ is a disjoint collection of manifolds each of which has the property that all points have the same isotropy group.)

Transversality lemma The map $f$ is $\varepsilon$-equivariantly homotopic to a transverse map. Further if $f$ is already transverse near a closed subset $C$ (invariant under $G$ ) then we can assume the homotopy fixed on $C$.

Proof Consider the conjugacy classes $\{G\}=\mathcal{C}_{1}, \mathcal{C}_{2}, \ldots, \mathcal{C}_{q}=\{\{e\}\}$ of subgroups of $G$ ordered such that $H \in \mathcal{C}_{i}, K \in \mathcal{C}_{j}, H \subset K$ imply $j<i$. Suppose that $f$ is transverse near $f^{-1}(0 \times X) \cap V^{H}$ for each $H \in \mathcal{C}_{i}, 1 \leq i<j$ and consider $H \in \mathcal{C}=\mathcal{C}_{j}$. Let $W=V^{H}$ and $U$ be as above and consider the open subset $V_{H} \subset W$. Denote by $V_{\mathcal{C}}$ the union $\cup_{H \in \mathcal{C}} V_{H}$ and by $W_{\mathcal{C}}$ the union $\cup_{H \in \mathcal{C}} V^{H}$. By supposition $f$ is already transverse near $W-V_{H}$ for each $H \in \mathcal{C}$ and this implies that $f^{-1}(0 \times X) \cap V_{\mathcal{C}}$ is a closed subset $C$ of $W_{\mathcal{C}}$.

We start by making $f$ semi-parallel near $C$. For any point $x \in V^{H}, H \in \mathcal{C}$ we can deform $f$ to be semi-parallel by choosing a small $\varepsilon$-neighbourhood $N$ of $0 \in U$, squeezing $f \mid N_{x}$ to $f(x)$ and growing $f \mid N$ radially outwards in $U_{f x}$ to be the identity $N_{x} \rightarrow N_{f x}$. By doing the same homotopy for each point in the orbit of $x$ (which is a discrete subset of $V_{\mathcal{C}}$ in bijection with the set of cosets $G / H)$ we have an equivariant homotopy which makes $f$ semi-parallel at $x$. A similar process will make $f$ semi-parallel near $x$ (work in a neighbourhood which is small enough not to meet any of its translates under $G / H$ ) and we can leave $f$ fixed near a closed subset where it is already semi-parallel. A standard patch-by-patch argument will now make $f$ semi-parallel near $C$.

We now apply ordinary transversality to $f: V_{\mathcal{C}} \times Q \rightarrow W_{\mathcal{C}} \times X$ to make it transverse to $0 \times X$ by a homotopy which leaves the $X$-coordinate fixed. Again we work patch by patch, copying the (small) homotopy around orbits, and preserve the semi-parallel condition by extending the movement by crossing with the identity on $N$ for a suitably small neighbourhood of 0 in $U$. The method of proof makes the relative statement clear. 
Continuing with the proof of theorem 1 , we observe that a representative of $\pi_{k} C_{V}(X)$ is a collection $T$ of parallel framed manifolds in $S^{k} \times W^{H}$ equivariantly labelled in $X$ for varying $H$, where parallel means having normal bundle whose fibres can be identified with the appropriate translate of $V$. Further, we may assume that these are smooth manifolds with boundary labelled by $*$. This uses non-degeneracy of the basepoint, which implies that $X$ is homotopy equivalent to $X$ with a whisker $\{*\} \times I$ attached at the basepoint. Deform the labelling map $l$ to be transverse to $* \times 1 / 2$ and then stretch $[0,1 / 2]$ to $[0,1]$; the proof of transversality needed here is a simplified version of the proof given above. Make $l \mid T^{H_{i}}$ transverse to $* \times 1 / 2$ by induction on $i$; each move is extended to a neighbourhood by moving normal fibres like their centres and the process then continues.

Now by the main transversality lemma, a representative of $\pi_{k} \Omega^{V} S^{V}(X)$ is a collection $U$ of semi-parallel framed manifolds. Notice that the argument just given implies that we may ignore the labelling map near $*$ and hence that we are mapping into $V \times X$ rather than $S^{V}(X)$. Moreover again by the same argument we can assume that these manifolds have boundaries labelled by $* \times 1 / 2$, which is then replaced by $*$. But $G$-connectivity of $X$ implies that all components of these manifolds can be assumed to have boundary. Choose a point $x \in U_{H}$ say and choose a small disc centre $x$, with collar both invariant under $H$ where small means that the disc does not meet any of its translates under representatives of $G / H$. Use connectivity of $X^{H}$ to deform the label on the disc to $*$ and extend using the collar and a path to the basepoint. Do the same for the translates. The interiors of the discs are now labelled by $*$ and can be deleted.

Now by the compression theorem such a framed manifold (with boundary) can be made parallel; the version of the compression theorem needed here is the codimension 0 (with boundary) version of the multi-compression theorem (see the note at the end of section 4 of [7]). The isotopy is not small in this version but of the form, shrink to the neighbourhood of a codimension 1 spine, then perform a small isotopy. This can be made equivariant by choosing an appropriate spine in the quotient manifold under the group action, using an equivariant shrink and then the above patch by patch argument.

\section{Proofs of theorems 2 and 3}

We shall describe a map $q: B C_{M \times \mathbb{R}}(X) \rightarrow C_{M}(S X)$ which we shall prove to be a homotopy equivalence; this proves theorem 3 . The description of $q$ will 
make it clear that the following diagram commutes up to homotopy:

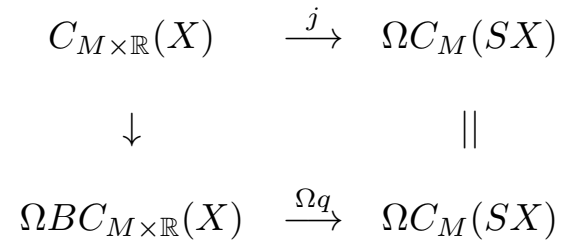

Here $j$ is defined in a similar way to $j_{V}$; we replace $C_{M \times \mathbb{R}}(X)$ by the homotopy equivalent space $C_{M \times \mathbb{R}}^{-}(X)$ of disjoint finite sets of "little 1-disks" parallel to $\mathbb{R}$ (little bi-collars) and use a similar formula to that for $j_{V}$ (above the statement of theorem 1). Below we give a precise definition of $B C_{M \times \mathbb{R}}$ in which $C_{M \times \mathbb{R}}$ is replaced by a strictly associative monoid $C$. The vertical unlabelled map in the diagram is then equivalent to the natural map $C \rightarrow \Omega B C$. Thus the homotopy equivalence $q$ is induced by $j$ and theorem 2 can be deduced by first applying theorem 3 with $M=V$ and then applying theorem 1 with $X$ replaced by $S X$.

\section{Description of $q$}

In order to define the classifying space $B C_{M \times \mathbb{R}}(X)$ we shall replace $C_{M \times \mathbb{R}}(X)$ by a homotopy equivalent strictly associative monoid and we do this in a standard way, cf [8; bottom of page 213]. We shall also use "little 1-disks" as above. To be precise, consider the associative monoid $C$ of which a point is a collection (configuration) of little 1-discs in $M \times(0, t)$ for some $t \geq 0$. The 1-discs are required to be parallel to $\mathbb{R}$ and the collection is closed under action of $G$ and equivariantly labelled in $X$. Addition is defined by juxtaposing the intervals $(0, t),\left(0, t^{\prime}\right)$ to form the interval $\left(0, t+t^{\prime}\right)$.

The classifying space $B=B C$ is the realisation of the simplicial space of which an $n$-simplex is an $n$-tuple of such configurations, with faces defined by omitting the first or last configuration or by adding an adjacent pair of configurations. We shall define $q: B \rightarrow C_{M}(S X)$.

First we require some technicalities on slices of the standard simplex.

Let $\Delta_{n}=\left\{\left\{x_{1}, \ldots, x_{n}\right\} \in \mathbb{R}^{n} \mid 0 \leq x_{n} \leq \cdots \leq x_{1} \leq 1\right\}$ denote the standard $n$ simplex. The $k^{\text {th }}$ slice of $\Delta_{n}$, denoted $L_{k}$ is the $(n-1)$-cell $\left\{x \in \Delta_{n} \mid x_{k}=\frac{1}{2}\right\}$. The vertices of $\Delta_{n}$ are $v_{0}=(0, \ldots, 0), v_{1}=(1,0, \ldots, 0), \cdots v_{n}=(1, \ldots, 1)$. Let $\sigma_{k}$ be the face of $\Delta_{n}$ spanned by $v_{0}, \cdots, v_{k-1}$ and let $\tau_{k}$ be the face spanned by $v_{k}, \cdots, v_{n}$. Then $\Delta_{n}$ is the join of $\sigma_{k}$ with $\tau_{k}$ and the product of $\sigma_{k}$ with $\tau_{k}$ can be identified with $L_{k}$ by $(u, v) \mapsto \frac{1}{2}(u+v)$. Given parameters $s_{k} \in(0, \infty)$ for $1 \leq k \leq n$ we will show how to thicken and pull apart the slices $L_{k}$ so that interiors of thickened slices are pairwise disjoint, see the figure. The thickened $k^{t h}$ slice $T L_{k}$ will be defined as the image of an embedding $S_{k}:\left(0, s_{k}\right) \times \sigma_{k} \times \tau_{k} \rightarrow \Delta_{n}$. 


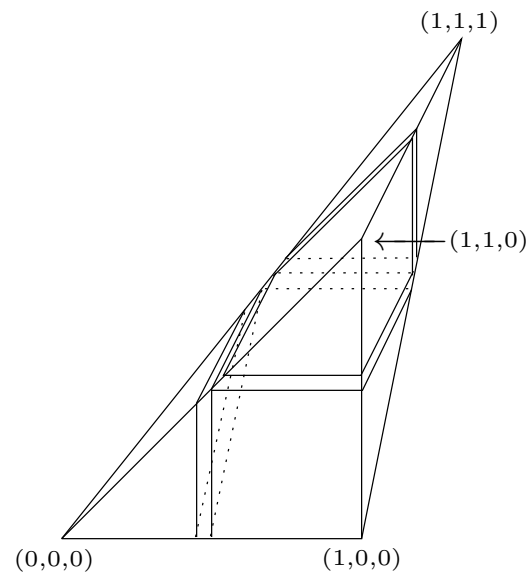

Figure : disjoint thickened slices of the standard 3-simplex

Let $\theta:[0, \infty) \rightarrow\left[\frac{1}{2}, 1\right)$ be the homeomorphism given by $\theta(t)=\frac{2}{\pi} \arctan (1+t)$. Let $v_{i} \in \sigma_{k}, v_{j} \in \tau_{k}$, so $i<k \leq j$. Define $S_{k}\left(t, v_{i}, v_{j}\right)=u_{i, k} v_{i}+\left(1-u_{i, k}\right) v_{j}$, where $u_{i, k}=\theta\left(s_{i+1}+\cdots+s_{k-1}+t\right)$, and in general define

$$
S_{k}(t, u, v)=\sum_{\substack{0 \leq i<k \\ k \leq j \leq n}} \lambda_{i} \mu_{j} S_{k}\left(t, v_{i}, v_{j}\right)
$$

where $u=\sum_{0 \leq i<k} \lambda_{i} v_{i}$ and $v=\sum_{k \leq j \leq n} \mu_{j} v_{j}$.

We are ready to define $q$. Consider configurations $c_{k}$ in $M \times\left(0, s_{k}\right)$, for $1 \leq k \leq$ $n$, and labelling functions $x_{k}: c_{k} \rightarrow X$. Each little 1-disc $d \in c_{k}$ is determined by its centre point $c(d)$ and the radius $\varepsilon(d)$ of the 1-disc. A typical point in an $n$-simplex of $B$ can be written as $b=\left[t_{1}, \cdots, t_{n},\left(c_{1}, x_{1}\right), \cdots,\left(c_{n}, x_{n}\right)\right]$. We will define $q(b)$. If $\left(t_{1}, \cdots, t_{n}\right)$ is not in the image of any $S_{k}$ then define $q(b)=*$. Suppose then that $\left(t_{1}, \cdots, t_{n}\right)=S_{k}(t, u, v)$. Let $d \in c_{k}$, then $c(d)=\left(c^{\prime}, c^{\prime \prime}\right) \in M \times\left(0, s_{k}\right)$ and we define $c^{\prime}$ to be in the configuration of $q(b)$ if $\left|t-c^{\prime \prime}\right| \leq \varepsilon(d)$. The label of $c^{\prime}$ in $S X$ has $X$-coordinate $x_{k}(d)$ and suspension coordinate $\frac{1}{2 \varepsilon(d)}\left(t+\varepsilon(d)-c^{\prime \prime}\right)$.

\section{Proof that $q$ is a homotopy equivalence}

We adapt the proof given in Segal [8] of the case when $M=\mathbb{R}^{n}$ and the action of $G$ is trivial (see [8; proposition 2.1]). We need to consider two other spaces. $B^{\prime}$ is the classifying space of the partial monoid of configurations in $M$ labelled in $X$, where the operation is union of disjoint configurations (see [8; page 215]) and $B^{\prime \prime}$ is the classifying space of the sub-partial monoid of $C \simeq C_{M \times \mathbb{R}}(X)$ (defined above) comprising configurations which project homeomorphically to $M$ with 
the operation being defined only for configurations which project disjointly to $M$. Projection $p: B^{\prime \prime} \rightarrow B^{\prime}$ is a homotopy equivalence with inverse induced by inclusion of $M$ as $M \times \frac{1}{2} \subset M \times(0,1)$. Further $q^{\prime}: B^{\prime} \rightarrow C_{M}(S X)$ can be defined by using the halfway slices $L_{k}$ and "full-size" bicollars and then $q^{\prime}: B^{\prime} \rightarrow C_{M}(S X)$ is a homeomorphism by the same proof as [8; proposition 2.3]. Thus it suffices to prove that the inclusion $i: B^{\prime \prime} \rightarrow B$ is a homotopy equivalence, and that the following diagram commutes up to homotopy:

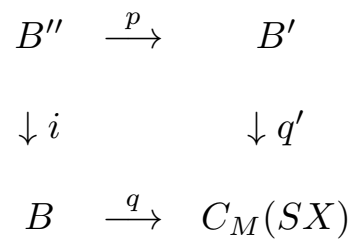

The following considerations explain why it is to be expected that $i$ : $B^{\prime \prime} \rightarrow B$ is a homotopy equivalence.

A thin $\mathbb{R}$-slice of a configuration in $M \times \mathbb{R}$ projects homeomorphically to $M$. Further the addition in $C$ shows that any configuration is the sum of such configurations. This implies that any 1 -simplex in $B$ can be deformed across a sequence of 2-simplexes until it is replaced by a chain of 1 -simplexes in $B^{\prime \prime}$. In a similar way, higher-dimensional simplexes in $B$ can be deformed into $B^{\prime \prime}$.

This argument can readily be sharpened to prove that $B^{\prime \prime} \rightarrow B$ is a weak homotopy equivalence. The proof given in [8] can be regarded as sharpening this argument to prove a full homotopy equivalence. In [8] the process of splitting configurations into $\mathbb{R}$-slices is made precise as "disintegration", see [8; pages 217-218]. On a more formal level, the proof in [8] carries over to the equivariant case with obvious changes. There is one comment which needs to be made. We need an equivariant version of the application of non-degeneracy of the basepoint made just below [8, proposition 2.7]. This is done by using a simple transversality argument as in the proof of theorem 1 above.

Finally to see that $q^{\prime} p \simeq q i$ observe that if $b \in B^{\prime \prime}$ is determined as above by an $n$-tuple of configurations of little 1-discs then $q^{\prime} p(b)$ is defined using the halfway slices and full-size bicollars whilst $q i(b)=q(b)$, defined above, uses slices near but not exactly halfway and with small bicollars. Thus the required homotopy is given by sliding the slices to halfway (use the formula for the slices above and a homotopy of $\theta$ to the constant map to $\frac{1}{2}$ ) and expanding the collars.

\section{Proof of theorem 4}

We shall need an extension of theorem 1 which applies to configurations in a general $G$-manifold. 
Let $M$ be a $G$-manifold of dimension $n$ without boundary and with tangent bundle $T_{M}$. Let $E_{M}$ be the sphere bundle in $T(M) \oplus 1$; this bundle can also be thought of as the disc bundle in $T(M)$ with each fibre of the sphere bundle squeezed to a point (which is taken as the basepoint in the fibre). Let $E_{M}(X)$ denote the bundle with fibre $S^{n}(X)$ obtained from $E_{M}$ by taking the smash product with $X$ in each fibre. Let $\Gamma_{M}(X)$ denote the space of equivariant sections of $E_{M}(X) . \Gamma_{M}(X)$ is based by the base section (given by the basepoint in each fibre).

There is a map $j: C_{M}(X) \rightarrow \Gamma_{M}(X)$ given by replacing $C_{M}(X)$ by the equivalent little disc space $C_{M}^{\circ}(X)$ and then using the description given in McDuff [4; page 95]. (McDuff calls this map $\phi_{\varepsilon}$; the formula is in the middle of the page.) Theorem 1 has the following generalisation:

Theorem 5 Let $X$ be a based $G$-connected space. Then $j: C_{M}(X) \rightarrow$ $\Gamma_{M}(X)$ is a weak homotopy equivalence.

Proof The proof is a straightforward generalisation of the proof of theorem 1. We first discuss the case when $G$ is trivial. An element of $\pi_{k} C_{M}(X)$ can be regarded as a compact submanifold of $\mathbb{R}^{k} \times M$ of dimension $k$, such that the projection on $\mathbb{R}^{k}$ is an immersion, and which is labelled in $X$ so that the boundary is labelled by $*$ (see the whisker argument used in the proof of theorem 1 here).

Now an element of $\pi_{k} \Gamma_{M}(X)$ can be interpreted by transversality to the zero section as a compact submanifold $W$ say of $\mathbb{R}^{k} \times M$ which is labelled in $X$ so that the boundary is labelled by $*$. Further the normal bundle pulls back from $T(M)$ and hence each fibre has an explicit identification with the tangent fibre to $M$ at the same point. Locally, using a local trivialisation of $T(M)$, this means that the normal bundle is framed with framing not necessarily the same as this local trivialisation. But the compression theorem can now be applied locally to make these framings agree and thus $W$ can locally be moved to a parallel submanifold. Using the relative version of the compression theorem and working patch by patch we can move $W$ globally to a parallel submanifold and this shows that $j_{*}: \pi_{k} C_{M}(X) \rightarrow \pi_{k} \Gamma_{M}(X)$ is surjective. A similar relative argument shows that $j_{*}$ is injective.

This proof is extended to the general case (when $G$ is non-trivial) in exactly the same way that the proof of the non-equivariant loops-suspension theorem given in [7; section 7] was extended to the equivariant case in the proof of theorem 1: we replace transversality by equivariant transversality and we replace the local moves given by the compression theorem by equivariant moves, by copying the local move around an orbit. 
We can now prove theorem 4. If $V$ has no non-trivial summand, we can write $C_{V}(X)$ as the product $X^{G} \times C_{Q \times \mathbb{R}}(X)$ where $Q$ is the unit sphere in $V$. This is because $0 \in V$ must be labelled by a fixed point of $G$ in $X$ and the configuration is described by giving the label at 0 (which might be $*$ ) and an equivariant configuration in $V-\{0\} \cong Q \times \mathbb{R}$. Write $A$ for $C_{Q \times \mathbb{R}}(X)$

Now by theorem 3 , there is a group completion $A \rightarrow \Omega C_{Q}(S X)$. Further by theorem $5 C_{Q}(S X)$ has the weak homotopy type of $\Gamma_{Q}(S X)$. Thus there is a weak group completion $A \rightarrow \Omega \Gamma_{Q}(S X)$.

Next consider $\Gamma_{Q}(S X)$. This is the space of cross-sections of $E_{Q}(S X)$, where $E_{Q}$ is the disk bundle of $T_{Q}$, with each boundary sphere squeezed to a point, and $E_{Q}(S X)$ is obtained by smashing each fibre with $S X$. Ignoring the $G-$ action, this is a bundle with fibre $S^{n-1} \wedge S^{1} \wedge X$ and, ignoring $X$ as well for the moment, we can identify each fibre $S^{n-1} \wedge S^{1}$ with $S^{V}$ : think of the $S^{n-1}$ in the fibre over $q \in Q$ as the subspace of $V$ parallel to the tangent plane to $Q$ at $q$, compactified at $\infty$; think of $S^{1}$ as the similarly compactified perpendicular line. It follows that we can identify each fibre of $E_{Q}(S X)$ with $S^{V}(X)$ and taking account of the $G$-action we see that $\Gamma_{Q}(S X)$ is the space of equivariant maps $Q \rightarrow S^{V}(X)$.

We can now interpret $\Omega \Gamma_{Q}(S X)$ as the subspace $Z$ of $\Omega^{V} S^{V}(X)$ comprising maps $S^{V} \rightarrow S^{V}(X)$ which near zero and infinity map to $*$. We shall construct a homotopy equivalence $u$ : $\Omega^{V} S^{V}(X) \rightarrow X^{G} \times Z$. The required weak homotopy equivalence $t$ (of the statement of theorem 4) is the composition of $u$ with the identification of $Z$ (up to weak homotopy type) with the group completion of $A$ which is described above. It is straightforward to check that $t \circ j_{V}$ is homotopic to the natural map $X^{G} \times A \rightarrow X^{G} \times Z$ and theorem 4 follows.

It remains to construct $u$. Consider an equivariant map $f: S^{V} \rightarrow S^{V}(X)$. Now $f$ must map $0 \in V$ to either the basepoint of $S^{V}(X)$ or to $0 \times p$ in $S^{V} \wedge X$ where $p \in X^{G}$ and $p \neq *$. Thus in either case $f$ determines a point of $X^{G}$. Further, by squeezing a neighbourhood of 0 to 0 and then radially expanding a small sphere near zero to infinity, we can homotope $f$ so that there is a small disc $D$ centred at 0 such that $f$ identifies $D / \partial D$ with $S^{V} \times p$. Further we can squeeze a neighbourhood of $\infty$ to $\infty$. Once this has been done, we can see that $f$ on $V-D$ determines a map in $Z$. These constructions define $u: \Omega^{V} S^{V}(X) \rightarrow X^{G} \times Z$. There is a map $v: X^{G} \times Z \rightarrow \Omega^{V} S^{V}(X)$ given by sending $(p, g)$ to the map which identifies $D / \partial D$ with $S^{V} \times p$ and maps $V-D$ by $g$. It is straightforward to check that $u$ and $v$ are inverse homotopy equivalences. 


\section{References}

[1] Shôrô Araki, Mitutaka Murayama, $G$-homotopy types of $G$-complexes and representations of $G$-cohomology theories, Publ. Res. Inst. Math. Sci. 14 (1978) 203-222

[2] H.Hauschild, Äquvariante Konfigurationsräume und Abbildungsräume, Springer Lecture Notes No. 788 (1979) 281-315

[3] U.Koschorke, B.Sanderson, Self-intersections and higher Hopf invariants, Topology, 17 (1978) 283-290

[4] Dusa McDuff, Configuration spaces of positive and negative particles, Topology, 14 (1975) 91-107

[5] J.P.May, The geometry of iterated loop spaces, Springer Lectures Notes Series, 271 Springer-Verlag (1972)

[6] R.J.Milgram, Iterated loop spaces, Annals of Math., 84 (1966) 386-403

[7] Colin Rourke, Brian Sanderson, The compression theorem, arxiv:math.GT/9712235

[8] Graeme Segal, Configuration spaces and iterated loop spaces, Invent. Math. 21 (1973) 213-221

[9] Graeme Segal, Some results in equivariant homotopy theory, preprint (1979) available from: http://www.maths.warwick.ac.uk/ $\mathrm{bjs} / \mathrm{segal} . \mathrm{htm}]$

[10] Stefan Waner, Equivariant homotopy theory and Milnor's theorem, Trans. Amer. Math. Soc. 258 (1980) 351-368 